\title{
ESTUDOS FARMACOLÓGICOS DO ÁCIDO LIPÓICO NAS ALTERAÇÕES COMPORTAMENTAIS E NA ATIVIDADE DA SUPERÓXIDO DISMUTASE NO CORPO ESTRIADO DE RATOS APÓS CONVULSÕES INDUZIDAS POR PILOCARPINA
}

\author{
Pharmacological effects of lipoic acud in behavioural alterations and in striatal \\ superoxide dismutas e activity of rats after pilocarpine - induced seizures \\ ${ }^{1}$ Rivelilson Mendes de Freitas \\ ${ }^{1}$ Professor Adjunto do Setor de Farmacologia da Universidade Federal do Piauí - UFPI. \\ Rua Cícero Eduardo, s/n, Junco, Picos, 64600-000, Piauí.
}

*Autor para correspondência e-mail: rivelilson@ufpi.br

Recebido em 19/02/2009 - Aceito em 28/02/2009

\begin{abstract}
RESUMO: A epilepsia do lobo temporal é um dos distúrbios neurológicos mais comuns e apresenta uma taxa de prevalência de $5 \%$. O presente estudo investigou as ações farmacológicas do ácido lipóico nas alterações comportamentais e na atividade da superóxido dismutase (SOD) no corpo estriado de ratos adultos (2 meses de idade) que apresentaram convulsão e estado epiléptico (EP), através da administração de pilocarpina (400mg/kg, i.p., P400). Os estudos comportamentais em animais adultos mostraram que a administração de pilocarpina produz sinais colinérgicos periféricos, movimentos estereotipados e tremores em todos os animais. Em 75\% dos animais houve o desenvolvimento de convulsões e EP. No mesmo grupo foi observada uma taxa de mortalidade de $60 \%$. Por sua vez, o prétratamento com ácido lipóico, 30 min antes da administração de pilocarpina produziu uma redução de $50 \%$ no índice de convulsão e EP $(p<0.05)$, e ainda reduziu a taxa de mortalidade em $50 \%(p<0,05)$. Com relação aos estudos neuroquímicos não foram observadas alterações significativas na atividade da SOD no corpo estriado de ratos adultos, no entanto, o pré-tratamento com ácido lipóico produziu um aumento significativo na atividade da SOD de $14 \% \quad(p<0.05)$. Nossos resultados indicam que as convulsões induzidas por pilocarpina apresentaram alterações comportamentais características da atividade epiléptica e pode não haver mudanças na atividade da SOD durante a fase aguda das convulsões. No entanto, pode-se sugerir que os efeitos protetores do ácido lipóico podem ser decorrentes da modulação da atividade desta enzima, na tentativa de proteger o animal contra o dano neuronal produzido pelo estresse oxidativo observado no corpo estriado de ratos. As alterações comportamentais e na atividade da SOD observada sugere uma extensa participação do corpo estriado de ratos adultos na propagação e/ou manutenção do processo convulsivo, e que drogas capazes de prevenir essas alterações podem apresentar um potencial anticonvulsivante.
\end{abstract}

PALAVRAS ChaVeS: Convulsões, Corpo estriado, Pilocarpina, Superóxido Dismutase, Estresse oxidativo.

ABSTRACT: The temporal lobe epilepsy is one of the most common neurological disorders and has a prevalence rate of $5 \%$. This study investigated the pharmacological actions of lipoic acid on behavioral changes and in the superoxide dismutase (SOD) activity in striatum of adult rats (2-months-old) who had seizures and status epilepticus (SE) through the administration of pilocarpine $(400 \mathrm{mg} / \mathrm{kg}$, i.p., P400). The behavioral studies in adults showed that the administration of pilocarpine produces peripheral cholinergic signs, tremors and stereotyped movements in all animals. In $75 \%$ of the animals was observed the development of seizures and SE. The same group was observed a mortality rate of $60 \%$. In turn, the pre- 
treatment with lipoic acid, $30 \mathrm{~min}$ before administration of pilocarpine produced a reduction of $50 \%$ in seizures and SE rates $(p<0.05)$, and reduced the mortality rate in $50 \%(p<0,05)$. The studies showed that the neurochemical of SOD activity in the period of $24 \mathrm{~h}$ is not significant changes in the striatum, however, the pre-treatment with lipoic acid produced a significant increase in SOD activity of $14 \%(p<0.05)$. Our results indicate that seizures induced by pilocarpine exhibit behavioral changes characteristic of the epileptic activity and may be no changes in activity of SOD. However, one can suggest that the protective effects of lipoic acid may be the result of modulation of the activity of this enzyme, in an attempt to protect the animal against neuronal damage produced by oxidative stress observed in the striatum of rats. Behavioral changes and the activity of SOD observed suggests an extensive participation of the striatum of adult rats in the propagation and/or maintenance of the convulsive process and that drugs capable of preventing these changes may present a potential anticonvulsant.

KEYWORDS: Seizures, Striatum, Pilocarpine, Superoxide Dismutase, Oxidative stress.

\section{INTRODUÇÃO}

A epilepsia do lobo temporal é um dos distúrbios neurológicos mais comuns e apresenta uma taxa de prevalência de 5\%. Pode ser definida como uma afecção crônica de etiologia diversa, caracterizada por crises repetidas, devido a uma descarga excessiva dos neurônios cerebrais em diferentes estruturas e associada eventualmente com diversas alterações comportamentais, podendo ocorrer também quando há um aumento do nível basal de excitação do Sistema Nervoso Central (SNC), superior ao limiar da convulsão (De LORENZO et al., 2001). As crises convulsivas podem ser associadas às diversas mudanças bioquímicas no corpo estriado afetando vários neurotransmissores (FREITAS et al., 2003a,b), e a atividade de enzimas antioxidantes, tais como a superóxido dismutase, catalase, glutationa peroxidase e glutationa reduzida, que podem estar envolvidas na fisiopatologia das convulsões límbicas (SIMONIC et al., 2000).

As convulsões induzidas por pilocarpina produzem mudanças neuroquímicas durante a sua instalação e nos fenômenos epileptogênicos de sua propagação e/ou manutenção (MARINHO et al., 1997), e também pode ser útil para caracterizar os mecanismos de ações de drogas anticonvulsivantes para o tratamento da epilepsia. O modelo de convulsão induzido por pilocarpina em animais é bastante utilizado para estudar a fisiopatologia do processo convulsivo, uma vez que, reproduz as alterações comportamentais e eletroencefalográficas que são semelhantes à epilepsia do lobo temporal de humanos (BEM-ARI et al., 1981). Este modelo pode também ser utilizado para estudar o envolvimento dos sistemas antioxidantes como moduladores da epileptogênese, como também permite observar as ações farmacológicas de drogas anticonvulsivantes nas alterações comportamentais e neuroquímicas relacionadas com a atividade epiléptica (PEREIRA et al., 2007).

Dentre as inúmeras mudanças neuroquímicas vistas durante a fase aguda do processo convulsivo têm sido verificada várias alterações na produção de lipídio peróxidos, no conteúdo do nitrito e nitrato, na concentração da glutationa reduzida (GSH), nas atividades enzimáticas da superóxido dismutase (SOD) e catalase (CAT). De um modo geral, os radicais livres têm sido implicados na patogênese de várias doenças neurológicas, incluindo a epilepsia. Os radicais livres podem produzir várias alterações neuroquímicas durante o estresse oxidativo, que tem sido visto no corpo estriado de ratos adultos durante as convulsões límbicas (FREITAS et al., 2004a,b). Os neurônios produzem EROs, tais como: o peróxido de hidrogênio e os radicais superóxido, hidroxila e óxido nítrico. Visto que estes participam de reações essenciais para o organismo, as EROs são constantemente formadas e removidas, no entanto, podem ser prejudiciais, quando sua produção é superior a remoção mediada pelos sistemas antioxidantes de defesa (JARRETT et al., 2008).

Os radicais livres são produzidos e removidos do organismo através de um rigoroso controle pelos sistemas de defesa antioxidantes. No entanto, quando há uma quebra entre a produção e a remoção, estas espécies reativas derivadas do oxigênio podem produzir vários efeitos neurotóxicos no sistema nervoso central (SNC). Diante do exposto, seria importante verificar a ação de enzimas antioxidantes, entre elas da SOD, que é responsável pela conversão do radical superóxido em peróxido de hidrogênio $\left(\mathrm{H}_{2} \mathrm{O}_{2}\right)$ (DEVI et al., 2008). $\mathrm{O} \mathrm{H}_{2} \mathrm{O}_{2}$ por si só não é um radical livre, mas pode através de reações óxido-redução converte-se em um potente radical livre. É sabido que o estresse oxidativo (EO), tem uma importante participação na neurogênese das convulsões. O cérebro é mais vulnerável que outros tecidos ao EO, por que além de conter uma grande quantidade de lipídios e metais oxidáveis, apresenta menos mecanismos de defesa antioxidante em relação aos outros tecidos (WALZ et al., 2000).

Em recente trabalho, foi sugerido que o dano neuronal produzido pelas convulsões induzidas pela pilocarpina pode ser resultante do aumento dos níveis de peroxidação lipídica e nitrito no corpo 
estriado de ratos convulsivos, da mesma, não foi evidenciado um aumento compensatório na atividade da superóxido dismutase nessa área durante a fase aguda das convulsões. Baseado, nesses argumentos o objetivo do presente estudo foi investigar as ações do ácido lipóico nas alterações comportamentais e na atividade da superóxido dismutase no corpo estriado de ratos adultos durante a fase aguda das convulsões induzidas pela pilocarpina.

\section{MATERIAL E MÉTODOS}

\section{Animais}

Foram utilizadas ratos Wistar machos, adultos (2 meses de idade, 250-280g), provenientes do Biotério Central do Departamento de Fisiologia e Farmacologia, da Universidade Federal do Piauí. Durante todos os experimentos, os animais foram mantidos em gaiolas de acrílico de $30 \times 30 \times 30 \mathrm{~cm}^{3}$ onde foram observados durante $24 \mathrm{~h}$ com no máximo 6 animais, em condições controladas (temperatura ambiente de $24-25^{\circ} \mathrm{C}$ e umidade de 50 a $60 \%$ ), com ciclo claro / escuro alternado de 12 horas (07:00 am a 07:00 pm), recebendo ração padrão tipo Purina e água ad libitum. Os experimentos foram realizados de acordo com o guia de cuidados e usos de animais de laboratório do Departamento de Saúde e Serviços Humanos dos Estados Unidos da América (EUA).

\section{Tratamentos}

Os animais foram tratados com pilocarpina ( $400 \mathrm{mg} / \mathrm{kg}$, i.p., $\mathrm{n}=18)$ e observados por 24 horas (P400) após a administração. Os animais controles foram tratados com solução salina ( $\mathrm{NaCl}, 0,9 \%$, $\mathrm{n}=14$, Controle). Um terceiro grupo de animais foi tratado com ácido lipóico (10mg/kg, i.p., $\mathrm{n}=12$ ) e 30 min após esse tratamento os animais foram tratados com pilocarpina (400 mg/kg, i.p., AL + pilocarpina). Outro grupo foi tratado somente com ácido lipóico (10mg/kg, i.p., $\mathrm{n}=12$, AL). Após 24 horas da administração de pilocarpina, os animais que apresentaram convulsões, estado epiléptico e sobreviveram ao tratamento foram sacrificados sem anestesia por decapitação em guilhotina (Havard, USA), e em seguida, seus cérebros foram removidos. O corpo estriado foi dissecado sobre gelo e armazenado em papel alumínio devidamente identificado, pesado e conservado a $-80^{\circ} \mathrm{C}$ para uso posterior. Os grupos controle, AL e AL + pilocarpina também foram sacrificados da mesma forma. As dosagens neuroquímicas da atividade da superóxido dismutase foram realizadas após $24 \mathrm{~h}$ da dissecação.

\section{Estudo comportamental}

Os animais tratados e controles foram divididos em gaiolas contendo no máximo 6 animais e colocados em ambiente reservado, sendo feita à observação direta. Todos os grupos experimentais foram observados após os tratamentos, de acordo com os protocolos experimentais, perfazendo um total de 24 horas de observação.

Os seguintes parâmetros foram observados: presença de sinais colinérgicos periféricos (SCP miose, piloereção, cromodacriorréia, salivação, diarréia e diurese), tremores, movimentos estereotipados (aumento da atividade de roer, coçar, mastigar e wet-dog shakes (ato de sacudir - semelhante a um cachorro molhado)), convulsões motoras (incluindo movimentos clônicos das extremidades superiores que ocorrem em aproximadamente 30 minutos após administração da pilocarpina), progredindo para o estado de mal epiléptico (caracterizado por convulsões motoras límbicas definidas como contínuas quando ocorrem por um período maior que 30 minutos) e ainda determinou-se o número de animais que morrem em cada grupo experimental. Depois desse período de observação, os animais foram sacrificados por decapitação e seus os cérebros removidos para a dissecação do corpo estriado para a realização dos estudos neuroquímicos.

\section{Determinação da atividade enzimática da Superóxido Dismutase (SOD)}

A atividade da SOD foi determinada através da técnica descrita anteriormente por FLOHÉ \& OTTING, 1984. Foi preparado o meio da reação contendo tampão fosfato de potássio $50 \mathrm{mM}, \mathrm{pH} 7,8$, xantina $500 \mu \mathrm{M}$; cianeto de potássio $200 \mu \mathrm{M}$; EDTA $1 \mathrm{mM}$. Os homogenatos $(10 \% \mathrm{p} / \mathrm{v})$ foram preparados com tampão fosfato de potássio $50 \mathrm{mM}, \mathrm{pH} 7,8$. Em seguida centrifugados a $15000 \mathrm{rpm}$ durante $15 \mathrm{~min}$ a $4{ }^{\circ} \mathrm{C}$. Os sobrenadantes foram removidos para determinação da atividade da SOD. A xantina oxidase (XO - $5 \mathrm{U} / \mathrm{mL})$ usada na reação foi preparada a partir da solução padrão de XO $(1 \mu \mathrm{L}$ para $80 \mu \mathrm{L}$ de tampão fosfato de potássio $50 \mathrm{mM}, \mathrm{pH} 7,8$ ). Em seguida foi realizado o ensaio contendo $975 \mu \mathrm{L}$ do meio 
reacional, $20 \mu \mathrm{L}$ do da amostra e $5 \mu \mathrm{L}$ da $\mathrm{XO}$, a mistura foi agitada e em seguida realizada a leitura a $550 \mathrm{~nm}$ durante 6 minutos. A leitura do branco cinético foi feita a $550 \mathrm{~nm}$. A quantidade da atividade da SOD das amostras foi calculada usando a média das absorções lineares obtidas durante 6 min pela curva. Os resultados foram expressos em $\mathrm{U} / \mathrm{mg}$ de proteína. A concentração da proteína foi obtida através do método descrito por Lowry e colaboradores (1951). Uma unidade (U) da atividade da SOD corresponde à inibição de $50 \%$ da reação do $\mathrm{O}_{2}{ }^{-}$com o citocromo $\mathrm{C}$.

\section{Análise Estatística}

Os resultados que obedeciam a uma distribuição paramétrica foram analisados por Análise de Variância (ANOVA) com teste de Student Newman Keuls (post hoc) pelo programa GraphPad Prism versão 3.00 para Windows, GraphPad Software, San Diego California USA. Copyright (c) 1994-1999 por GraphPad software. Os dados não paramétricos (percentagens) foram analisados pelo mesmo programa utilizando o teste do qui-quadrado. O mesmo programa (GraphPad Prism@) foi utilizado para confecção do gráfico apresentado neste trabalho. As diferenças foram consideradas estatisticamente significativas a partir de $p<0,05$.

\section{RESULTADOS}

Os estudos comportamentais foram realizados como descrito anteriormente. Todos os animais adultos tratados com pilocarpina $(400 \mathrm{mg} / \mathrm{kg}$, sc; $n=18)$, durante $24 \mathrm{~h}$ de observação, apresentaram sinais colinérgicos periféricos (SCP), tais como, miose, piloereção, cromodacriorréia, salivação, diarréia, diurese, e também movimentos estereotipados (ME), envolvendo o aumento da atividade de roer, coçar, mastigar e wet-dog shakes (ato de sacudir - semelhante a um cachorro molhado) e tremores. As convulsões ocorreram em 75\% (14) que apareceram nos 25 minutos iniciais, sendo instalado o estado de mal epiléptico, em 75\% (14) dos animais. Foi observado um índice de sobrevivência de $40 \%$ (7) nesse grupo.

O pré-tratamento com ácido lipóico, 30 min antes da administração de pilocarpina produziu as alterações comportamentais descritas na tabela 1. Nenhuma alteração nos índices de SCP, ME e tremores observados durante os estudos comportamentais no grupo P400 foi modificada pelo prétratamento com o ácido lipóico. Entretanto, nesse grupo $(A L+P 400)$ foi observada uma redução de forma significativa de $50 \%$ nos índices das convulsões e do estado epiléptico, como também se verificou uma redução na taxa de mortalidade em 50\% em comparação ao grupo P400 (Tabela 1). Nenhum dos animais tratados somente com solução salina $0,9 \%$ ou ácido lipóico apresentaram alterações comportamentais características das convulsões induzidas por pilocarpina.

Tabela 1 - Efeito de pré-tratamento com ácido lipóico nas alterações comportamentais de ratos adultos durante as convulsões induzidas por pilocarpina.

\begin{tabular}{ccccccc}
\hline Grupos & $\begin{array}{c}S C P \\
(\%)\end{array}$ & $\begin{array}{c}\text { ME } \\
(\%)\end{array}$ & $\begin{array}{c}\text { Tremores } \\
(\%)\end{array}$ & $\begin{array}{c}\text { Convulsões } \\
(\%)\end{array}$ & $\begin{array}{c}\text { Estado epiléptico } \\
(\%)\end{array}$ & $\begin{array}{c}\text { TM } \\
(\%)\end{array}$ \\
\hline P400 & 100 & 100 & 100 & 75 & 75 & 60 \\
AL + P400 & 100 & 100 & 100 & $25^{\mathrm{a}}$ & $25^{\mathrm{a}}$ & $10^{\mathrm{a}}$ \\
\hline
\end{tabular}

O grupo P400 foi tratado com pilocarpina (400 mg/kg, i.p. $n=18$; P400). $\mathrm{O}$ grupo $A L+P 400$ foi prétratado com ácido lipóico (10mg/kg, i.p.), e $30 \mathrm{~min}$ após recebeu pilocarpina (400 mg/kg, i.p. $n=12 ; A L+$ P400). Os animais controles foram tratados com salina $0,9 \%$ (i.p., $n=14$ ). Outro grupo foi tratado somente com ácido lipóico (10mg/kg, i.p., LA, $n=12)$. Após os tratamentos os animais foram observados durante 24h para a determinação dos sinais colinérgicos periféricos (SCP), movimentos estereotipados (ME), tremores, convulsões, estado epiléptico, e a taxa de mortalidade (TM) de cada grupo. Os resultados dos estudos comportamentais foram expressos em percentagem. a quando comparado ao grupo P400 ( $p<0,05$; Teste do Qui-quadrado).

Os resultados dos efeitos do ácido lipóico na atividade da SOD no corpo estriado de ratos adultos após convulsões induzidas por pilocarpina foram apresentados na figura 1. No grupo P400 não foi verificada nenhuma alteração significativa na atividade da SOD em relação ao grupo controle (controle $=2,38 \pm 0,14$; pilocarpina $=2,41 \pm 0,18$ ), $[\mathrm{p}=\mathrm{N}$.S.] no corpo estriado dos ratos adultos. Por sua vez, o pré-tratamento com ácido lipóico, $30 \mathrm{~min}$ antes da administração de pilocarpina produziu um 
aumento significativo na atividade enzimática da SOD de $14 \%$ quando comparado ao grupo pilocarpina (pilocarpina $=2,41 \pm 0,18 ; \mathrm{AL}+\mathrm{P} 400=2,75 \pm 0,14),[\mathrm{p}<0.0025]$ (Figura 1). Nenhuma alteração significativa na atividade da SOD no grupo tratado somente com ácido lipóico não foi verificada em comparação aos valores dos grupos controle (controle $=2,38 \pm 0,14 ; A L=2,29 \pm 0,15$ ) $[p=N . S$.] e P400 $(A L=2,29 \pm 0,15$; pilocarpina $=2,41 \pm 0,18)[p=N . S$.], no corpo estriado dos ratos adultos (Figura 1 ).

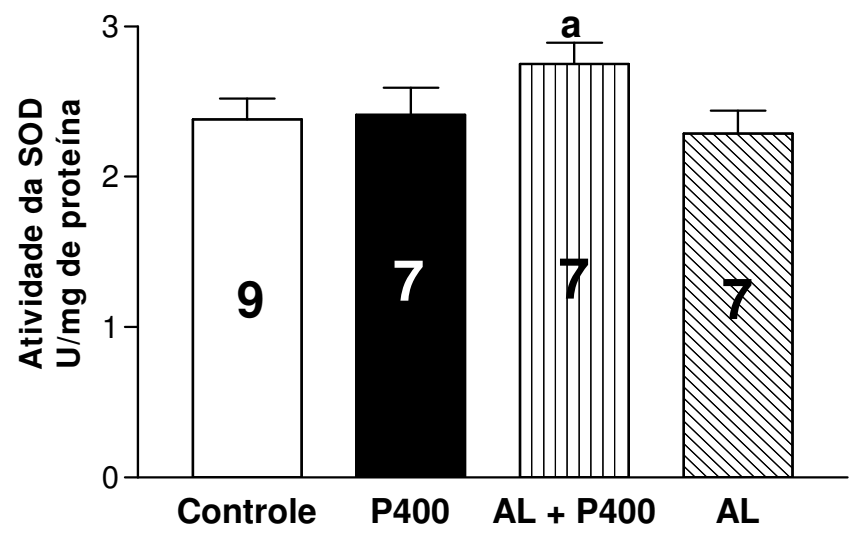

Figura 1 - Efeitos do ácido lipóico na atividade da superóxido dismutase (SOD) no corpo estriado de ratos adultos após convulsões induzidas por pilocarpina.

O grupo $\mathrm{P} 400$ foi tratado com pilocarpina (400 mg/kg, i.p. $n=18$; $\mathrm{P} 400)$. $\mathrm{O}$ grupo $\mathrm{AL}+\mathrm{P} 400$ foi prétratado com ácido lipóico (10mg/kg, i.p.), e $30 \mathrm{~min}$ após recebeu pilocarpina (400 mg/kg, i.p. $n=12 ; A L+$ P400). Os animais controles foram tratados com salina $0,9 \%$ (i.p., $n=14$ ). Outro grupo foi tratado somente com ácido lipóico (10mg/kg, i.p., LA, $\mathrm{n}=12)$. Os animais estudados apresentaram convulsão, estado epiléptico e foram sacrificados $24 \mathrm{~h}$ após os tratamentos. Os valores representam à média \pm EPM do número de experimentos inseridos nas barras. Para análise estatística foram usados ANOVA e teste $t$ Student Newman Keuls como post hoc. ${ }^{a}$ quando comparado ao grupo P400 $(p<0,05)$.

As convulsões límbicas induzidas pela pilocarpina em animais reproduzem alterações comportamentais e eletroencefalográficas que são semelhantes à epilepsia do lobo temporal de humanos (CAVALHEIRO et al., 1991). O modelo de convulsão induzido por pilocarpina foi utilizado para investigar as ações farmacológicas do ácido lipóico nos parâmetros comportamentais e na atividade da enzima SOD no corpo estriado de ratos adultos durante a fase aguda do processo convulsivo. Imediatamente após a administração de pilocarpina em nossos experimentos, os ratos apresentaram persistentes alterações comportamentais, incluindo piloereção, cromodacriorréia, acinesia, ataxia, tremores, automatismos mastigatórios como mioclonia dos músculos faciais, e movimentos de cachorro molhado, que persistem de 10 a 15 minutos. Estas alterações comportamentais são contínuas até a instalação das convulsões motoras límbicas, incluindo movimentos clônicos das extremidades superiores que ocorreram em $75 \%$ dos animais em aproximadamente 30 minutos após administração da pilocarpina. No mesmo grupo as convulsões progrediram até o desenvolvimento do estado epiléptico em $75 \%$ destes. Nesse mesmo grupo foi observada uma taxa de sobrevivência de $40 \%$.

Nossos resultados quanto às alterações comportamentais durante as convulsões estão de acordo com as observações descritas anteriormente por outros estudos (TURSKI et al., 1983; CAVALHEIRO et al., 1994; MARINHO et al., 1998; SANTOS et al., 2008). No presente estudo, nós demonstramos que administração sistêmica de ácido lipóico reduz o número de animais que apresentam convulsões e EP induzido por pilocarpina, e também aumenta o índice de sobrevivência dos animais. Isto foi evidenciado pelas mudanças comportamentais observadas em animais que receberam o estímulo convulsivo. Os resultados sugerem que o ácido lipóico pode apresentar uma importante ação anticonvulsivante no modelo de convulsão induzido por pilocarpina. Entretanto, não foi observada nenhuma mudança nos parâmetros (SCP, ME e tremores). Essas alterações podem ser vistas durante as convulsões e são decorrentes da estimulação dos receptores colinérgicos periféricos $M_{3}$. Esses achados sugerem que a ação do ácido lipóico pode não ser mediada através do bloqueio destes receptores muscarínicos, uma vez que este composto antioxidante apresentou importantes efeitos anticonvulsivantes. Sendo, assim, mais estudos com relação aos outros sistemas de neurotransmissores envolvidos na atividade epiléptica devem ser realizados para esclarecer o mecanismo de ação anticonvulsivante do ácido lipóico. 
Alguns estudos procuram esclarecer à fisiopatologia do processo convulsivo, procurando detectar as mudanças em nível neuroquímico (SIMONIC et al., 2000) em áreas envolvidas com a epileptogênese das convulsões, a fim de contribuir para o pronto esclarecimento dos mecanismos da atividade epiléptica. Além das alterações cerebrais durante as crises convulsivas, também tem sido investigada a ação de compostos antioxidantes (vitamina C e E) (BARROS et al., 2007; XAVIER et al., 2007) como potenciais tratamentos anticonvulsivantes. Esses estudos visam deslumbrar o papel dos antioxidantes no processo convulsivo. Diante disso, e na tentativa de esclarecer a ação neuroprotetora e anticonvulsivante de antioxidantes exógenos como ácido lipóico, faz-se necessário investigar a atividade da enzima (SOD), que não se encontra alterada durante o estresse oxidativo cerebral no animal convulsivo. Esta enzima é a principal defesa antioxidante cerebral e o radical livre removido pela sua atividade, o superóxido $\left(\mathrm{O}_{2}{ }^{-}\right)$, está implicado na patogênese de várias doenças neurológicas, inclusive nas convulsões límbicas, reforçando o nosso interesse em identificar sua ação durante as crises convulsivas e após o tratamento com drogas antioxidantes. Este radical pode ser gerado no cérebro através de vários mecanismos (ineficiência do transporte de elétrons durante o ciclo de Krebs nas mitocôndrias, metabolização das monoaminas e aminoácidos, reação enzimática da xantina oxidase, e através do metabolismo do ácido araquidônico). Esse radical pode ser removido pela ação da superóxido dismutase (NAFFAH-MAZZACORATTI et al., 2001), portanto, drogas capazes de modular positivamente a atividade desta poderiam reduzir os efeitos neurotóxicos mediados pelos radicais superóxidos durante as crises convulsivas.

Investigou-se, assim, os efeitos do ácido lipóico na atividade da SOD no corpo estriado durante a fase aguda da convulsão induzida por pilocarpina. Não foi verificada alteração significativa na atividade da SOD na área cerebral investigada, sugerindo que esta pode não ser capaz de evitar os danos neuronais observados no corpo estriado de ratos convulsivos (MARINHO et al., 1997), acredita-se, assim, que os radicais livres produzidos nessa região cerebral podem ser essenciais para a propagação e/ou manutenção das convulsões mediadas pelo estriado. Por sua vez, o pré-tratamento com ácido lipóico aumentou a atividade enzimática estriatal de forma significativa. As mudanças na atividade enzimática, nos experimentos sugerem que durante o processo convulsivo, pode não haver interferência na atividade da enzima como um mecanismo sinérgico de propagação das convulsões, por outro lado o ácido lipóico pode ter sido capaz de reduzir o número de animais que apresentaram convulsões e que progrediram para o EP, bem como aumentaram a taxa de sobrevivência destes, através da remoção de radicais livres produzidos durante as crises convulsivas através do aumento da atividade da SOD estriatal. Entretanto, não foi observada nenhuma mudança na atividade da SOD nos animais tratados somente com o ácido lipóico, reforçando a hipótese de seu mecanismo de ação anticonvulsivante ser mediado pela modulação da atividade de enzimas antioxidantes.

Estudos sugerem que o ácido lipóico produz seus efeitos através da remoção de radicais hidroxilas e da inibição da oxidação de lipídios e proteínas (BIST e BHATT, 2009). Devido ao seu potente efeito antioxidante seria também capaz de evitar o dano neuronal ocasionado pelas espécies reativas derivadas do oxigênio produzidas durante as doenças neurodegenerativas (MACZUREK et al., 2008). Também foi descrito uma importante ação para esse composto antioxidante contra o processo inflamatório cerebral induzido por radicais livres (BIEWENGA et al., 1997). Devido aos seus efeitos farmacológicos proeminentes durante o estresse oxidativo e na modulação enzimática da SOD verificada em nossos experimentos, sugere-se que esta droga antioxidante poderá servir no futuro após a realização de estudos que esclareçam completamente o seu mecanismo de ação, como um potencial agente terapêutico neuroprotetor e anticonvulsivante para o tratamento da epilepsia em humanos.

\section{CONCLUSÕES}

Nossos resultados indicam que as convulsões induzidas por pilocarpina apresentam alterações comportamentais características da atividade epiléptica e pode não induzir mudanças na atividade da SOD durante a fase aguda no corpo estriado de ratos adultos. Porém, o pré-tratamento com o ácido lipóico aumentou notoriamente a atividade da SOD estriatal, esses achados sugerem que pode haver a participação dos radicais livres produzidos no corpo estriado durante a propagação e/ou manutenção do processo convulsivo induzido por pilocarpina. $O$ ácido lipóico apresentou importante efeito anticonvulsivante, uma vez que é capaz de reduzir o número de animais que convulsionam e aumenta a taxa de sobrevivência destes ao processo convulsivo.

Novos estudos serão realizados para uma melhor avaliação dos efeitos do ácido lipóico em outros sistemas antioxidantes e de neurotransmissão (dopaminérgico, noradrenérgico, serotoninérgico, GABAérgico e glutamatérgico), com o propósito de contribuir para o esclarecimento das ações anticonvulsivantes e neuroprotetoras do ácido lipóico durante as convulsões límbicas induzidas pela pilocarpina. 


\section{REFERÊNCIAS BIBLIOGRÁFICAS}

BARROS, D. O. et al. Effects of the vitamin $E$ in catalase activities in hippocampus after status epilepticus induced by pilocarpine in Wistar rats. Neuroscience Letters. v. 416, p. 227-230, 2007.

BEN-ARI, Y. et al. Electrographic, clinical and pathological alterations following systemic administration of kainic acid, bicuculine, deoxyglucose or pentylenetetrazole: metabolite mapping using the deoxyglucose method with special reference to the pathology of epilepsy. Neuroscience. v. 6, p. 1361-1391, 1981.

BIEWENGA, G. P.; HAENEN, G. R. M. M.; BAST, A. The pharmacology of the antioxidante lipoic acid. General Pharmacology. v. 29, p. 315-331, 1997.

BIST, R.; BHATT, D. K. The evaluation of effect of alpha-lipoic acid and vitamin e on the lipid peroxidation, gamma-amino butyric and serotonin level in the brain of mice (Mus musculus) acutely intoxicated with lindane. Journal of the Neurological Sciences. v. 276, p. 99-102, 2009.

CAVALHEIRO, E. A. et al. Long-term effects of pilocarpine in rats: structural damage of the brain triggers kindling and spontaneous recurrent seizures. Epilepsia. v. 32, p. 778-782, 1991.

CAVALHEIRO, E. A. et al.Spontaneous recurrent seizures in rats: amino acid and monoamine determination in the hippocampus. Epilepsia. v. 35, p. 1-11, 1994.

De LORENZO, R.J. et al. Long-term alteration of calcium homeostastic mechanisms in the pilocarpine model of temporal lobe epilepsy. Brain Research. v. 903, p. 1-12, 2001.

DEVI P. U.; MANOCHA, A., VOHORA, D. Seizures, antiepileptics, antioxidants and oxidative stress: an insight for researchers. Expert Opinion on Pharmacotherapy. v. 9, p. 3169-3177, 2008.

FREITAS, R. M. et al. Acute alterations of neurotransmitters levels in striatum of young rat after pilocarpine-induced status epilepticus. Arquivos de Neuro-Psiquiatria., v. 61, p. 430-433, 2003a.

FREITAS, R. M. et al. Pilocarpine-induced seizures in adult rats: monoamine content and muscarinic and dopaminergic receptor changes in the striatum. Comparative Biochemistry and Physiology C: Pharmacology. Toxicol. Endocrinol., v. 136, n. 2, p. 103-108, 2003b.

FREITAS, R. M. et al. Pilocarpine-induced seizures in adult rats: lipid peroxidation level, nitrite formation, GABAergic and glutamatergic receptor alterations in the hippocampus, striatum and frontal córtex. Pharmacology Biochemistry Behavior. v. 78, n. 2, p. 327-332, 2004a.

FREITAS, R. M. et al. Catalase activity in cerebellum, hippocampus, frontal cortex and striatum after status epilepticus induced by pilocarpine in Wistar rats. Neuroscience Letters. v. 365, n. 2, p. 102-105, 2004b.

FLOHE, L.; OTTING, F. Superoxide dismutase assays. Methods Enzymology. v. 105, p. 93-104, 1984.

JARRETT, S. G., et al. Mitochondrial DNA damage and impaired base excision repair during epileptogenesis. Neurobiology of Disease. v. 30, p. 130-138, 2008.

LOWRY, O.H. et al. Protein measurement with follin phenol reagent. Journal Biological Chemistry. v. 193, p. 265-275, 1951.

MACZUREK, A. et al. Lipoic acid as an anti-inflammatory and neuroprotective treatment for Alzheimer's disease. Advanced Drug Delivery Reviews. v. 60, p. 1463-1470, 2008.

MARINHO, M. M. F. et al. Inhibitory action of a calcium channel blocker (nimodipine) on seizures and brain damage induced by pilocarpine and lithium-pilocarpine in rats. Neuroscience Letters. v. 235, p. 1316, 1997. 
MARINHO, M. M. F. et al. Effects of lithium, alone or associated with pilocarpine, on muscarinic and dopaminergic receptors and on phosphoinositide metabolism in rat hippocampus and striatum. Neurochemisty International. v. 33, p. 299-306, 1998.

NAFFAH-MAZZACORATTI, M. G. et al. Superoxide dismutase, glutathione peroxidase activities and the hydroperoxide concentration are modified in the hippocampus of epileptic rats. Epilepsy Research. v. 46, p. 121-128, 2001.

PEREIRA, M. B. et al. Study pharmacologic of the GABAergic and glutamatergic drugs on seizures and status epilepticus induced by pilocarpine in adult Wistar rats. Neuroscience Letters. v. 419, p. 253-257, 2007.

SANTOS, L. F. et al. Neuroprotective actions of vitamin C related to decreased lipid peroxidation and increased catalase activity in adult rats after pilocarpine-induced seizures. Pharmacology Biochemistry and Behavior. v. 89, p. 1-5, 2008.

SIMONIC, A. et al. plus pilocarpine induced status epilepticus - biochemical changes. Neuroscience Research. v. 36, p. 157-166, 2000.

TURSKI, W. A. et al. Limbic seizures produced by pilocarpine in rats: a behavioural, electroencephalographic and neuropathological study. Behavior Brain Research. v. 9, p. 315-335, 1983.

XAVIER, S. M. L. et al. Vitamin C antioxidant in hippocampus of adult Wistar rats after seizures and status epilepticus induced by pilocarpine, Neuroscience Letters. v. 420, p. 76-79, 2007.

WALZ, R. et al. F. Lipid peroxidation in hippocampus early and late after status epilepticus induced by pilocarpine of kainic acid in Wistar rats. Neuroscience Letters. v. 291, p. 179-182, 2000. 